\title{
GAUSS CURVATURE OF GAUSSIAN IMAGE OF MINIMAL SURFACES
}

\author{
By LI HAIZHONG
}

\begin{abstract}
In this paper, we estimate the Gauss curvature of Gaussian image of minimal surfaces in $R^{n}(c)$, which equality case is exceptıonal mınımal surfaces in $R^{4}(c)$ defined by Johnson.
\end{abstract}

1. Introduction. Let $R^{n}(c)$ be an $n$-dimensional simply connected space form of constant curvature $c$. When $c>0, R^{n}(c)=S^{n}(c)$ when $c=0, R^{n}(c)=R^{n}$; when $c<0, R^{n}(c)=H^{n}(c)$. Let $M$ be a minimal surface in $R^{n}(c)$, we denote by $K(\leqq c)$ the Gauss curvature of $M$ with respect to the induced metric $d s_{M}^{2}$. On $M$, we choose a local field of orthonormal frames $e_{1}, \cdots, e_{n}$ in $R^{n}(c)$ in such a way that when restricted to $\mathrm{M}, e_{1}$ and $e_{2}$ are tangent to $M$ and $e_{3}, \cdots, e_{n}$ are normal to $M$. Their dual forms are $\omega_{1}, \cdots, \omega_{n}$. The metric of $\mathrm{M}$ is $d s_{M}^{2}=$ $\left.\left(\omega_{1}\right)^{2}+\omega_{2}\right)^{2}$. We consider Obata's Gauss map from $M$ to the space of all totally geodesic 2-subspaces in $R^{n}(c)$ ([8]). Riemannian metric of Gauss map $g(M)$ is $([8])$

$$
g^{*}\left(d s_{G}^{2}\right)_{\imath, a}^{=} \Sigma\left(\omega_{i \alpha}\right)^{2}=(c-K) d s_{M}^{2}
$$

which is degenerate at points where $K=c([8])$. Let $\bar{K}_{G}$ denote the Gauss curvature of $M$ with respect to $g^{*}\left(d s_{G}^{2}\right)$, which is the Gauss curvature of the Gaussian image of $M$.

When $n=3$, we have the following well-known result

THEOREM 1.1 (see Lawson [7]). Let $M$ be a minimal surface in $R^{3}(c)$ and $c-K \neq 0$ on $M$. Then

$$
\bar{K}_{\bar{G}}-1-\frac{c}{c-K}
$$

When $n \geqq 4$, the following result is well-known

THEOREM 1.2 (see $[1,5]$ ). Let $M$ be a minimal surface in $R^{n}$. Then

Received February 10, 1992, Revised June 22, 1992. 


$$
\bar{K}_{G} \leqq 2,
$$

and $\bar{K}_{G} \equiv 2$ on $M$ if and only if $M$ is a complex curve in $C^{2}$.

In this paper, our purpose is to generalize above Theorem 1.2 to minimal surfaces in $R^{n}(c)$. Our main result is the following theorem Then

THEOREM 1.3. Let $M$ be a minimal surface in $R^{n}(c)$ and $c-K \neq 0$ on $M$.

$$
\left.\bar{K}_{G} \leqq\right\urcorner-\frac{c}{c-K},
$$

and equality holds in (1.4) on $M$ if and only if $n=4$ and

$$
|A|^{4}=K_{N},
$$

where $|A|^{2}=\sum_{\alpha, \imath, j}\left(h_{\imath j}^{\alpha}\right)^{2}$ is the square length of the second fundamental form of $M$ and $K_{N}=\sum_{\alpha, \beta, \imath, j} R_{\alpha \beta i j}^{2}$ is the normal scalar curvature of $M$ in $R^{4}(c)$, i.e., $M$ is an exceptional minimal surface in $R^{4}(c)$ defined by Johnson in [6].

Remark 1.1. According to definition of exceptional minimal surface ([6]), minimal immersions of the 2-sohere $S^{2}$ into $R^{4}(c)$ are always exceptional (Chern [3]) (these surfaces are called "superminimal" by Bryant [2]). Thus, by Theorem 1.3 , these surfaces satisfy $\overline{K_{G}} \equiv 2-c /(c-K)$. We also note that notion of exceptional minimal surfaces in $R^{4}(c)$ is equivalent to $R$-surfaces by Y. C. Wong ([13]).

2. Fundamental lemmas. We need the following lemmas to prove Theorem 1.3 .

LEMMA 2.1. Let $M$ be a minimal surface in $R^{n}(c)$, then

$$
\left|\nabla\left(|A|^{2}\right)\right|^{2} \leqq 2|A|^{2}|\nabla A|^{2} .
$$

// equality holds in (2.1), then we have

$$
h_{111}^{\alpha}=h_{112}^{\alpha}=0, \quad \alpha \geqq 5 .
$$

Proof. Let $\mathrm{M}$ be a minimal surface $R^{n}(c)$. It is an elementary observation that at each point the dimension of the image of the second fundamental form $A$ of minimal surface $M$ is at most 2. Thus we may choose $e_{3}, \cdots, e_{n}$ so that $h_{\imath j}^{\alpha}=0$ for all $i, j$ and $\alpha \geqq 5$, i, e., we may choose the basis $e_{1}, e_{2}, \cdots, e_{n}$ so that the component $h_{\imath \jmath}^{\alpha}$ of $A$ satisfy ([11])

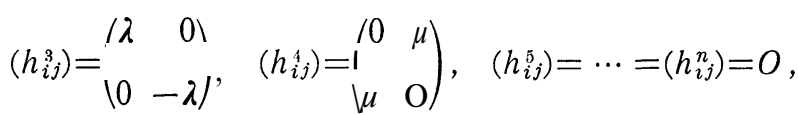


for some functions $\lambda$ and $\mu$. Let $|A|^{2}=\sum_{\alpha, \imath, j}\left(h_{i j}^{\alpha}\right)$ be the square length of the second fundamental form of $M$ and $K_{N}=\sum_{\alpha, \beta, i, j} R_{\alpha \beta i}^{2}$, be the normal scalar curvature of M. By (2.2) and Ricci equation we easily check that $|A|^{2}=$ $2\left(\lambda^{2}+\mu^{2}\right), K_{N}=16 \lambda^{2} \mu^{2}$.

Noting $\Sigma_{k}\left(h_{11 k}^{\alpha}\right)^{2}=\Sigma_{k}\left(h_{12 k}^{\alpha}\right)^{2}, 3 \leqq \alpha \leqq n$, by (2.2), we have

$$
\begin{aligned}
\left|\nabla\left(|A|^{2}\right)\right|^{2} & =4 \sum_{k}\left(\sum_{i, j, \alpha} h_{i j}^{\alpha} h_{i j k}^{\alpha}\right)^{2} \\
& =16 \sum_{k}\left(\lambda h_{11 k}^{3}+\mu h_{12 k}^{4}\right)^{2} \\
& \leqq 16 \sum_{k}\left(\lambda^{2}+\mu^{2}\right)\left[\left(h_{11 k}^{3}\right)^{2}+\left(h_{12 k}^{4}\right)^{2}\right] \\
& =8|A|^{2} \sum_{k}\left[\left(h_{11 k}^{3}\right)^{2}+\left(h_{11 k}^{4}\right)^{2}\right] .
\end{aligned}
$$

On the other hand, we have

$$
\begin{aligned}
|\nabla A|^{2} & =2 \sum_{\imath, k, \alpha}\left(h_{i i k}^{\alpha}\right)^{2}=4 \sum_{k, \alpha}\left(h_{11 k}^{\alpha}\right)^{2} \\
& \geqq 4 \sum_{k}\left[\left(h_{11 k}^{3}\right)^{2}+\left(h_{11 k}^{4}\right)^{2}\right] .
\end{aligned}
$$

We get (2.1) from (2.3) and (2.4).

If equality holds in (2.1), then we know that equality holds in (2.4). Noting that equality holds in (2.4) if and only if $h_{111}^{\alpha}=h_{112}^{\alpha}=0, \alpha \geqq 5$. Thus we have proved that if equality holds in (2.1), then we have $(2.1)^{\prime}$. We complete the proof of lemma 2.1 .

LEMMA 2.2. Let $M$ be a minimal surface in $R^{n}(c)$, then

$$
\begin{aligned}
\frac{1}{2} \Delta\left(|A|^{2}\right) & =|\nabla A|^{2}+2 c|A|^{2}-\frac{3}{2}|A|^{4}+2\left(\lambda^{2}-\mu^{2}\right)^{2} \\
& \geqq|\nabla A|^{2}+2 c|A|^{2}-\frac{3}{2}|A|^{4},
\end{aligned}
$$

and equality holds in (2.5) if and only if (1.5) holds, i.e., the following geometric condition makes sense

$$
\lambda= \pm \mu
$$

Proof. Denote the matrix $\left(h_{i j}^{\alpha}\right)$ by $H_{\alpha}, 3 \leqq \alpha \leqq n$. By Gauss-Codazzi-Ricci equations it was shown in [12] that

$$
\begin{aligned}
\frac{1}{2} \Delta\left(|A|^{2}\right)= & \sum_{\alpha, i, j, k}\left(h_{i j k}^{\alpha}\right)^{2}+\sum_{\alpha, \imath, j, k, l} h_{i j_{k}}^{\alpha}\left(h_{k l}^{\alpha} R_{l \imath j k}+h_{l i}^{\alpha} R_{l k j k}\right) \\
& +\sum_{a, \beta, \imath, j, k} h_{i j}^{\alpha} h_{k i}^{\beta} R_{\beta \alpha j k} \\
= & |\nabla A|^{2}+\sum_{a, \beta} \operatorname{tr}\left(H_{\alpha} H_{\beta}-H_{\beta} H_{\alpha}\right)^{2}-\sum_{a, \beta}\left(\operatorname{tr}\left(H_{\alpha} H_{\beta}\right)\right)^{2}+2 c|A|^{2} .
\end{aligned}
$$

By (2.2), it is easy to check the following formulas 


$$
\sum_{a, \beta} \operatorname{tr}\left(H_{\alpha} H_{\beta}-H_{\beta} H_{\alpha}\right)^{2}=-16 \lambda^{2} \mu^{2},
$$

$$
\sum_{\alpha, \beta}\left(\operatorname{tr}\left(H_{\alpha} H_{\beta}\right)\right)^{2}=4\left(\lambda^{4}+\mu^{4}\right) .
$$

Substituting (2.7) and (2.8) into (2.6), we get

$$
\begin{aligned}
\frac{1}{2} \Delta\left(|A|^{2}\right) & =|\nabla A|^{2}+2 c|A|^{2}-8\left(\lambda^{2}+\mu^{2}\right)^{2}+4\left(\lambda^{4}+\mu^{4}\right) \\
& =|\nabla A|^{2}+2 c|A|^{2}-\frac{3}{L^{n}} \mid A^{4}+2\left(\lambda^{2}-\mu^{2}\right)^{2} .
\end{aligned}
$$

From (2.9), equality holds in (2.5) if and only if $\lambda^{2}=\mu^{2}$, i. e., $\lambda= \pm \mu$, i. e., $|A|^{4}=K_{N}$. We complete the proof of lemma 2.2 .

LEMMA 2.3. (Otsuki [10] or see Ogata [9]). Let $M$ be a minimal surface in $R^{n}(c)$. If $|A|^{2} \neq 0, K_{N} \neq 0$ and $h_{111}^{\alpha}=h_{112}^{\alpha}=0,(\alpha \geqq 5)$ on $\mathrm{M}$. Then there is a 4dimensional totally geodesic submanifold of $R^{n}(c)$ such that $M$ is contained in the submanifold.

Remark 2.1. In [9], scalar fields $K_{(2)}$ and $N_{(2)}$ are defined by

$$
\begin{gathered}
K_{(2)}=\sum_{\alpha}\left[\left(h_{11}^{\alpha}\right)^{2}+\left(h_{12}^{\alpha}\right)^{2}\right], \\
N_{(2)}=\sum_{\alpha}\left(h_{11}^{\alpha}\right)^{2} \sum_{\alpha}\left(h_{12}^{\alpha}\right)^{2}-\left(\sum_{\alpha} h_{11}^{\alpha} h_{12}^{\alpha}\right)^{2} .
\end{gathered}
$$

Obviously, by (2.2), $|A|^{2}=2 K_{(2)}=2\left(\lambda^{2}+\mu^{2}\right), K_{N}=16 N_{(2)}=16 \lambda^{2} \mu^{2}$.

3. Proof of Theorem 1.3. Let $\mathrm{M}$ be a minimal surface in $R^{n}(c)$ and $c-K \neq 0$ on $\mathrm{M}$, where $K$ is the Gauss curvature of $M$ with respect to the induced metric $d s_{M}^{2}$. We choose the basis $e_{1}, \cdots, e_{n}$ such that we have (2.2). Let $\sigma=c-K=|A|^{2} / 2>0$, as well known, the Gauss curvature $\bar{K}_{G}$ of the conformal metric $\sigma d s_{M}^{2}$ satisfies (see [4])

$$
-\sigma \bar{K}_{G}=\sigma-c+\frac{1}{2} \frac{\Delta \sigma}{\sigma}-\frac{|\nabla \sigma|^{2}}{2 \sigma^{2}} .
$$

By use of lemma 2.1 and lemma 2.2,

$$
\begin{aligned}
\frac{1}{2} \Delta \sigma & =\frac{1}{4} \Delta\left(|A|^{2}\right)=\frac{1}{2}|\nabla A|^{2}+c|A|^{2}-\frac{3}{4}|A|^{4}+\left(\lambda^{2}-\mu^{2}\right)^{2} \\
& \geqq \frac{1}{2} \frac{|\nabla \sigma|^{2}}{\sigma}-3 \sigma^{2}+2 \sigma c .
\end{aligned}
$$

Combining (3.1) with (3.2), we obtain (1.4).

If equality holds in (1.4) on $\mathrm{M}$, then equality holds in (3.2) on $\mathrm{M}$, i.e., equalities hold in (2.1) and (2.5) on M. Thus we have $(2.1)^{\prime}$ and $(2.5)^{\prime}$ on $\mathrm{M}$. Combining this with lemma 2.3, we have $n=4$ and $|A|^{4}=K_{N}$ (that is $\lambda= \pm \mu$ ) on $\mathrm{M}$, i. e., $\mathrm{M}$ is an exceptional minimal surface in $R^{4}(c)$ defined by Johnson in [6]. 
Let $M$ be an exceptional minimal surface in $R^{4}(c)$, i. e., $|A|^{4}=K_{N}$, i. e., $\lambda= \pm \mu$ on $M$. In this case, by $\lambda^{2}=\mu^{2}=|A|^{2} / 4$, we know that $\lambda$ and $\mu$ are smooth functions on $M$. Equalities hold in (2.3) and (2.4) by a direct check, thus equality holds in (2.1). Combining this with $(2.5)^{\prime}$, we have proved that equality holds in (1.4) from (3.1) and (3.2). We complete the proof of Theorem 1.3 .

Acknowledgement. This paper is a part of author's doctoral thesis entitled "Curvature pinching theorems for minimal submanifolds" in department of mathematics of Sarajevo university, in 1992. He expresses his thanks to Prof. Muharem Avdispahic, Prof. Neda Bokan and Prof. Sun Zhenzu for their encouragements and teachings. Many thanks also to the referee for his useful suggestions and comments.

\title{
REFERENCES
}

[ 1 ] BARBOSA, J.L. AND Do CARMO, M., Stability of minimal surfaces and eigenvalues of the Laplacian, Math. Z., 173 (1980), 13-28.

[2] BRYANT, R.L., Conformal and minimal immersions of compact surfaces into the 4-sphere, J. Diff. Geom., 17 (1982), 455-473.

[ 3 ] CHERN, S. S., On the minimal immersions of the two-sphere in a space of constant curvature, Problems in Analysis, Princeton, 1970, 27-40.

[4] GOLDBERG, S.I., Curvature and Homology, Academic Press, New York, 1962.

[5 ] HOFFMAN, D. AND OSSERMAN, R., The geometry of the generalized Gauss map, Mem. Amer. Math. Soc., 236 (1980).

[6] JOHNSON, G.D., An intrinsic characterization of a class of minimal surfaces in constant curvature manifolds, Pacific J. Math., 149 (1991), 113-125.

[7] LAWSON, H.B., Complete minimal surfaces in $S^{3}$, Ann. of Math., 92 (1970), 335374.

[8] OBATA, M., The Gauss map of immersions of Riemannian manifolds in spaces of constant curvature, J. Diff. Geom., 2 (1968), 217-223.

[9] OGATA, T., Minimal surfaces in a sphere with Gaussian curvature not less than 1/6, Tohoku Math. J., 37 (1985), 553-560.

[10] OTSUKI, T., Minimal submanifolds with $m$-index 2 and generalized Veronese surfaces, J. Math. Soc. Japan, 24 (1972), 89-122.

[11] SAKAKI, M., Remarks on the rigidity and stability of minimal submanifolds, Proc. Amer. Math. Soc., 106 (1989), 793-795.

[12] SIMONS, J., Minimal varieties in Riemannian manifolds, Ann. of Math., 88 (1968), 62-105.

[13] WONG, Y.C., Contributions to the theory of surfaces in a 4-space of constant curvature, Trans. Amer. Math. Soc., 59 (1946), 467-507.

\author{
DEPARTMENT OF MATHEMATICS \\ ZHENGZHOU UNIVERSITY \\ ZHENGZHOU 450052 \\ PEOPLE'S REPUBLIC OF CHINA
}

